VOL. 21, No. 2 (2017), 1-13

\title{
On topological BE-algebras
}

\author{
S. Mehrshad AND J. Golzarpoor
}

ABstract. In this paper, we study some properties of uniform topology and topological BE-algebras and compare this topologies.

\section{INTRODUCTION}

The study of BCK/BCI-algebras was initiated by K. Iséki as a generalization of the concept of set-theoretic difference and propositional calculus([3],[4]). In [9], J. Neggeres and H. S. Kim introduced the notion of d-algebras which is a generalization of BCK-algebras. Moreover, Y. B. Jun, E. H. Roh and H. S. Kim [6] introduced a new notion, called BH-algebras, which is a generalization of BCK/BCI-algebras. Recently, as another generalization of BCK-algebras, the notion of BE-algebras was introduced by H. S. Kim and Y. H. Kim [7].

In section 3 we study some properties of uniform topology. In section 4 we study some general properties of topological BE-algebras, and finally in section 5 we obtain some relationships between this topologies.

\section{PRELIMINARIES}

Recall that a set $X$ with a family $\tau=\{U\}$ of its subsets is called a topological space, denoted by $(X, \tau)$, if $X, \emptyset \in \tau$, the intersection of any finite number of members of $\tau$ is in $\tau$ and the arbitrary union of members of $\tau$ is in $\tau$. The members of $\tau$ is called open sets of $X$. The complement $X \backslash U$ of an open set $U$ is said to be closed set. If $B$ is a subset of $X$, the smallest closed set containing $B$ is called the closure of $B$ and denoted by $\bar{B}$ (or $c l_{\tau} B$ ). A subset $P$ of $X$ is said to be a neighborhood of $x \in X$, if there exists an open set $U$ such that $x \in U \subseteq P$.

A subfamily $\left\{U_{\alpha}\right\}$ of $\tau$ is said to be a base of $\tau$ if for each $x \in U \in \tau$ there exists an $\alpha$ such that $x \in U_{\alpha} \subseteq U$, or equivalently, each $U$ in $\tau$ is the union of members of $\left\{U_{\alpha}\right\}$. A subfamily $\left\{U_{\beta}\right\}$ of $\tau$ is said to form a subbase for $\tau$ if the family of finite intersections of members of $\left\{U_{\beta}\right\}$ forms a base of $\tau$.

2000 Mathematics Subject Classification. Primary: 40B05; Secondary: 33E99.

Key words and phrases. BE-algebra, Filter, Uniform topology, Topological BEalgebra. 
Let $(X, \tau)$ be a topological

space. We have the following separation axioms in $(X, \tau)$ :

$\mathbf{T}_{\mathbf{0}}$ : For each $x, y \in X$ and $x \neq y$, there is at least one in an open neighborhood excluding the other.

$\mathbf{T}_{\mathbf{1}}$ : For each $x, y \in X$ and $x \neq y$, each has an open neighborhood not containing the other.

$\mathbf{T}_{\mathbf{2}}$ : For each $x, y \in X$ and $x \neq y$, both have disjoint open neighborhoods $U, V$ such that $x \in U$ and $y \in V$.

A topological space satisfying $T_{i}$ is called a $T_{i}$-space. A $T_{2}$-space is also known as a Hausdorff space.

Definition 2.1. Let $(X, *)$ be an algebra of type 2 and $\tau$ be a topology on $A$. Then $\mathcal{X}=(X, *, \tau)$ is called a

(i) Left (right) topological algebra, if for all $a$ in $X$ the map $X \hookrightarrow X$ is defined by $x \hookrightarrow a * x(x \hookrightarrow x * a)$ is continuous, or equivalently, if for any $x$ in $X$ and any open set $U$ of $a * x(x * a)$, there exists an open set $V$ of $x$ such that $a * V \subseteq U(V * a \subseteq U)$.

(ii) Semitopological algebra, or operation $*$ is separately continuous, if $X$ is right and left topological algebra.

(iii) Topological algebra, if the operation $*$ is continuous, or equivalently, if for any $x, y$ in $X$ and any open set(neighborhood) $W$ of $x * y$ there exist two open sets(neighborhoods) $U$ and $V$ of $x$ and $y$, respectively, such that $U * V \in W$.

Let $X$ be a nonempty set and $U, V$ be any subsets of $X \times X$. Define $U \circ V=\{(x, y) \in X \times X:(x, z) \in U$ and $(z, y) \in V$, for some $z \in X\}$, $U^{-1}=\{(y, x):(x, y) \in U\}, \triangle=\{(x, x): x \in X\}$.

Definition $2.2([5])$. By an uniformity on $X$ we shall mean a nonempty collection $\mathcal{K}$ of subsets of $X \times X$ which satisfies the following conditions:

(i) $\triangle \subseteq U$, for any $U \in \mathcal{K}$,

(ii) if $U \in \mathcal{K}$, then $U^{-1} \in \mathcal{K}$,

(iii) if $U \in \mathcal{K}$, then there exist $V \in \mathcal{K}$ such that $V \circ V \subseteq U$,

(iv) if $U, V \in \mathcal{K}$ then $U \cap V \in \mathcal{K}$,

(v) if $U \in \mathcal{K}$ and $U \subseteq V \subseteq X \times X$, then $V \in \mathcal{K}$.

The pair $(X, \mathcal{K})$ is called a uniform structure (uniform space).

Let $x \in X$ and $U \in \mathcal{K}$. Define $U[x]=\{y \in X:(x, y) \in U\}$.

Definition 2.3 ([11]). A BE-algebra is an algebra $(X, *, 1)$ of type $(2,0)$ such that satisfying the following axioms:

(1) $x * x=1$ for all $x \in X$,

(2) $x * 1=1$ for all $x \in X$,

(3) $1 * x=x$ for all $x \in X$,

(4) $x *(y * z)=y *(x * z)$, for all $x, y, z \in X$. 
A relation $\leq$ on $X$ is defined by $x \leq y$ if and only if $x * y=1$. If $X$ is a BE-algebra and $x, y \in X$, then $x *(y * x)=1$.

Definition 2.4 ([11]). We say that a BE-algebra $X$ is commutative if $(x *$ $y) * y=(y * x) * x$ for all $x, y \in X$.

Proposition 2.1 ([1]). Let $X$ be a commutative BE-algebra and $x, y, z \in X$. Then,

(5) $x * y=y * x=1 \Rightarrow x=y$,

(6) $(x * y) *((y * z) *(x * z))=1$.

Definition 2.5 ([11]). We say that a BE-algebra $X$ is transitive if $(y * z) \leq$ $(x * y) *(x * z)$ for all $x, y, z \in X$.

Proposition 2.2 ([11]). If $X$ is a commutative BE-algebra, then it is transitive.

Definition 2.6 ([11]). Let $A$ be a BE-algebra. A filter is a nonempty set $F \subseteq X$ such that for all $x, y \in A$

(i) $1 \in F$,

(ii) $x \in F$ and $x * y \in F$ imply $y \in F$.

Let $F$ be a filter in $X$. If $x \in F$ and $x \leq y$ then $y \in F$.

Definition 2.7 ([11]). A filter $F$ of a BE-algebra $X$ is said to be normal if it satisfies the following condition:

$$
x * y \in F \Rightarrow[(z * x) *(z * y) \in F \text { and }(y * z) *(x * z) \in F]
$$

for all $x, y, z \in X$.

Proposition 2.3 ([11]). If $X$ is a transitive BE-algebra, then every filter of $X$ is normal.

Proposition 2.4 ([11]). Let $F$ be a normal filter of a BE-algebra $X$. Define

$$
x \equiv{ }^{F} y \Leftrightarrow x * y, y * x \in F .
$$

Then

$(i) \equiv^{F}$ is a congruence relation on $X$, i.e., it is a equivalence relation on $X$ such that for each $a, b, c, d \in X$ if $a \equiv^{F} b$ and $c \equiv^{F} d$, then $a * c \equiv^{F} b * d$.

(ii) Let $\frac{x}{F}=\left\{y \in x: x \equiv{ }^{F} y\right\}$ be an equivalence class of $x$ and $\frac{X}{F}=\left\{\frac{x}{F}\right.$ : $x \in X\}$. Then $\frac{X}{F}$ is a BE-algebra under the binary operations given by:

$$
\frac{x}{F} * \frac{y}{F}=\frac{x * y}{F} .
$$

Definition $2.8([2])$. Let $X$ be a BE-algebra. If there exists an element 0 satisfying $0 \leq x$ (or $0 * x=1$ ) for all $x \in X$, then $X$ is called a bounded BE-algebra.

Notation. From now, in this paper $(X, *, 1)$ is a commutative BE-algebra. 


\section{UNIFORM TOPOLOGY ON BE-ALGEBRAS}

Theorem 3.1 ([8]). Let $\Lambda$ be an arbitrary family of filters of a BE-algebra $X$ which is closed under intersection. If $U_{F}=\left\{(x, y) \in X \times X: x \equiv{ }^{F} y\right\}$ and $\mathcal{K}^{*}=\left\{U_{F}: F \in \Lambda\right\}$, then $\mathcal{K}^{*}$ satisfies in the conditions $(i) \sim(i v)$ of Definition 2.2.

Theorem 3.2 ([8]). Let $\mathcal{K}=\left\{U \subseteq X \times X: U_{F} \subseteq U\right.$, for some $\left.U_{F} \in \mathcal{K}^{*}\right\}$. Then the pair $(X, \mathcal{K})$ is an uniform structure.

Theorem 3.3 ([8]). Given a BE-algebra $X$, then

$$
\tau=\{G \in X: \forall x \in G \exists U \in \mathcal{K} \text { s.t. } U[x] \subseteq G\}
$$

is a topology on $X$.

Definition 3.1. Let $(X, \mathcal{K})$ be an uniform structure. Then the topology $\tau$ is called an uniform topology on $X$ induced by $\mathcal{K}$.

We denote the uniform topology obtained by a family $\Lambda$, by $\tau_{\Lambda}$ and if $\Lambda=$ $\{F\}$, then we denote it by $\tau_{F}$.

Note that for any $x \in X, U[x]$ is an open neighborhood of $x$.

Theorem 3.4 ([8]). The pair $\left(X, \tau_{\Lambda}\right)$ is a topological BE-algebra.

Notation. Let $\Lambda$ be a family of filters of a BE-algebra $X$ which is closed under intersection and $F \in \Lambda$ and $A \subseteq X$. Then we define $U_{F}[A]=\bigcup_{a \in A} U_{F}[a]$.

Theorem 3.5. Let $\Lambda$ be a family of filters of a BE-algebra $X$ which is closed under intersection and $F \in \Lambda$ and $A \subseteq X$. Then the closure of $A$ is $\bigcap\left\{U_{F}[A]: U_{F} \in \mathcal{K}^{*}\right\}$ and it is denoted by $\bar{A}$ in the topological space $\left(X, \tau_{\Lambda}\right)$.

Proof. Let $x \in \bar{A}$. Then $U_{F}[x]$ is an open neighborhood of $x$ and we have $U_{F}[x] \cap A \neq \emptyset$, for all $F \in \Lambda$. Hence there exists $y \in A$ such that $y \in U_{F}[x]$. Hence $(x, y) \in U_{F}$ for all $F \in \Lambda$. Thus $x \in U_{F}[y] \subseteq U_{F}[A]$ for all $F \in \Lambda$. Conversely, let $x \in U_{F}[A]$ for all $F \in \Lambda$. Then there exists $y \in A$ such that $x \in U_{F}[y]$ and so $U_{F}[x] \cap A \neq \emptyset$ for all $F \in \Lambda$. Therefore $x \in \bar{A}$.

Theorem 3.6. Let $\Lambda$ be a family of filters of a BE-algebra $X$ which is closed under intersection, $K$ be a compact subset of $X$ and $W$ be an open set containing $K$. Then $K \subseteq U_{F}[K] \subseteq W$.

Proof. Since $W$ is an open set containing $K$, for each $k \in K$ we have $U_{F_{k}}[k] \subseteq$ $W$ for some $F_{k} \in \Lambda$. Hence $K \subseteq \bigcup_{k \in K} U_{F_{k}}[k] \subseteq W$. Since $K$ is a compact subset of $X$, there exist $k_{1}, k_{2}, \ldots, k_{n}$ such that

$$
K \subseteq U_{F_{k_{1}}}\left[k_{1}\right] \cup U_{F_{k_{2}}}\left[k_{2}\right] \cup \cdots \cup U_{F_{k_{n}}}\left[k_{n}\right] .
$$

Put $F=\bigcap_{i=1}^{n} F_{k_{i}}$. We claim that $U_{F}[K] \subseteq W$ for each $k \in K$. Let $k \in K$. Then there exists $1 \leq i \leq n$ such that $k \in U_{F_{K_{i}}}\left[K_{i}\right]$ and hence $k \equiv{ }^{F_{K_{i}}} k_{i}$. Now, let $y \in U_{F}[k]$, then $y \equiv{ }^{F} k$. Therefore we have $y \equiv{ }^{F_{K_{i}}} k_{i}$ and hence $y \in U_{F_{K_{i}}}\left[K_{i}\right] \subseteq W$. Hence $U_{F}[k] \subseteq W$ for any $k \in K$. Thus $K \subseteq U_{F}[K] \subseteq W$. 
Theorem 3.7. Let $\Lambda$ be a family of filters of a BE-algebra $X$ which is closed under intersection, $K$ be a compact subset of $X$ and $C$ be a closed subset of $X$. If $K \cap C=\emptyset$, then $U_{F}[K] \cap U_{F}[C]=\emptyset$ for some $F \in \Lambda$.

Proof. Since $K \cap C=\emptyset$ and $C$ is closed, $X \backslash C$ is an open set containing $K$. By Theorem 3.6 there exists $F \in \Lambda$ such that $U_{F}[K] \subseteq X \backslash C$. If $U_{F}[K] \cap U_{F}[C] \neq \emptyset$, then there exists $y \in X$ such that $y \in U_{F}[k]$ and $y \in U_{F}[c]$ for some $k \in K$ and $c \in C$, respectively. Hence $k \equiv^{F} c$ and then $c \in U_{F}[k] \subseteq U_{F}[K]$. This contradicts to the fact that $U_{F}[K] \subseteq X \backslash C$. Hence $U_{F}[K] \cap U_{F}[C]=\emptyset$.

\section{Topological BE-Algebras}

Theorem 4.1. Let $\mathcal{F}$ be a family of filters in a BE-algebra $X$ such that for each $F_{1}, F_{2} \in \mathcal{F}$, there exists $F_{3} \in \mathcal{F}$ such that $F_{3} \subseteq F_{1} \cap F_{2}$. Then there is a topology $\tau$ on $X$ such that $(X, *, \tau)$ is a topological BE-algebra.

Proof. Define $\tau=\{U \subseteq X: \forall x \in U \exists F \in \mathcal{F}$ s.t. $x / F \subseteq U\}$. For each $x \in X$ and $F \in \mathcal{F}$, the set $x / F \in \tau$, because if $y$ is an arbitrary element of $x / F$ then $y / F \subseteq x / F$. It is easy to see that $\tau$ is a topology on $X$. We prove that $*$ is continuous. For this, suppose $x * y \in U \in \tau$. Then for some $F \in \mathcal{F}, \frac{x * y}{F} \subseteq U$. Now, $x / F$ and $y / F$ are two open neighborhoods of $x$ and $y$, respectively, such that $x / F * y / F \subseteq \frac{x * y}{F} \subseteq U$.

Example 4.1. Let $X=\{a, b, c, d, 1\}$. Define a binary operation $*$ on $X$ as follow:

\begin{tabular}{l|lllll}
$*$ & 1 & $a$ & $b$ & $c$ & $d$ \\
\hline 1 & 1 & $a$ & $b$ & $c$ & $d$ \\
$a$ & 1 & 1 & $b$ & $c$ & $d$ \\
$b$ & 1 & $a$ & 1 & $c$ & $c$ \\
$c$ & 1 & 1 & $b$ & 1 & $b$ \\
$d$ & 1 & 1 & 1 & 1 & 1
\end{tabular}

Easily we can check that $(X, *, 1)$ is a BE-algebra [9]. Let $\tau=\{\{1, a, c\},\{b, d\}, X, \emptyset\}$. Then $(X, *, \tau)$ is a topological BE-algebra [8].

Theorem 4.2. Let $(X, *, \tau)$ be a topological BE-algebra.

(i) $(X, \tau)$ is discrete if and only if $\{1\}$ is open.

(ii) $(X, \tau)$ is Hausdorff if and only if $\{1\}$ is closed.

Proof. (i) Let $\{1\}$ be an open subset of $X$. Then by (1), $x * x=1 \in$ $\{1\}$ for all $x \in X$. Since $(X, *, \tau)$ is a topological BE-algebra, there exist neighborhoods $U$ and $V$ of $x$ such that $U * V \subseteq\{1\}$. Put $W=U \cap V$. Then $1=x * x \in W * W \subseteq U * V \subseteq\{1\}$ and so $W * W=\{1\}$. We claim that $W=\{x\}$. Let $y \in W$. Then $x * y \in W * W=\{1\}$ and $y * x \in W * W=\{1\}$. Hence $x=y$ and so $W=\{x\}$. The converse is trivial.

(ii) Suppose that $(X, *, \tau)$ is a Hausdorff space. We show that $X \backslash\{1\}$ is an open subset of $X$. Let $x \in X \backslash\{1\}$. Then $x \neq 1$. Hence there exist 
neighborhoods $U$ of $x$ and $V$ of 1 such that $U \cap V=\emptyset$. Thus $1 \notin U$. Therefore $x \in U \subseteq X \backslash\{1\}$ and so $X \backslash\{1\}$ is an open subset of $X$.

Conversely, let $\{1\}$ be closed and $x, y \in X$ such that $x \neq y$. Then $x * y \neq 1$ or $y * x \neq 1$. Let $x * y \neq 1$. Then $x * y \in X \backslash\{1\}$. Since $X \backslash\{1\}$ is open, there exist neighborhoods $U$ of $x$ and $V$ of $y$ such that $U * V \subseteq X \backslash\{1\}$. We claim that $U \cap V=\emptyset$. Let $U \cap V \neq \emptyset$. Let $y \in U \cap V$. Hence $1=y * y \in U \cap V \subseteq X \backslash\{1\}$ which is a contradiction. Therefore $(X, \tau)$ is a Hausdorff space.

Theorem 4.3. Let $(X, *, \tau)$ be a topological BE-algebra. Then the following are equivalent:

(i) $(X, \tau)$ is a Hausdorff space,

(ii) $(X, \tau)$ is $T_{1}$,

(iii) $(X, \tau)$ is $T_{0}$.

Proof. $(i) \Rightarrow($ ii $)$ and $(i i) \Rightarrow($ iii $)$ are clear.

$($ iii $) \Rightarrow(i)$ Let $x, y \in X$ and $x \neq y$. Then $x * y \neq 1$ or $y * x \neq 1$. Let $x * y \neq 1$. Since $X$ is a $T_{0}$ space, there is an neighborhood $U$ of $x * y$ such that $1 \notin U$. Since $(X, *, \tau)$ is a topological BE-algebra, there exist neighborhoods $V$ of $x$ and $W$ of $y$ such that $V * W \subseteq U$. We claim that $V \cap W=\emptyset$. Let $V \cap W \neq \emptyset$. Let $z \in V \cap W$. Hence $1=z * z \in V * W \subseteq U$. This is a contradiction. Hence $(X, \tau)$ is a Hausdorff space.

Theorem 4.4. Let $(X, *, \tau)$ be a topological BE-algebra and $F$ be a filter of $X$. Then 1 is an interior point of $F$ if and only if $F$ is open.

Proof. Suppose that 1 is an interior point of $F$. Then there exists a neighborhood $U$ of 1 such that $U \subseteq F$. Let $x \in F$ be an arbitrary element. Since $x * x=1$, there exist neighborhoods $V, W$ of $x$ such that $V * W \subseteq U \subseteq F$. Now, for each $y \in W$, we have $x * y \in F$. Since $F$ is a filter and $x \in F$, we have $y \in F$. Hence $W \subseteq F$ and so $F$ is open. The converse is trivial.

Theorem 4.5. Let $(X, *, \tau)$ be a topological BE-algebra and $F$ be a filter of $X$. If $F$ is open, then $F$ is closed.

Proof. Let $F$ be a filter of $X$ which is open in $(X, \tau)$. We show that $X \backslash F$ is open. Let $x \in X \backslash F$. Since $F$ is open, by Theorem 4.4, 1 is an interior point of $F$. Hence there exists a neighborhood $U$ of 1 such that $U \subseteq F$. Since $x * x=1$, there exist neighborhoods $V$ and $W$ of $x$ such that $V * W \subseteq U \subseteq F$. We claim that $V \subseteq X \backslash F$. Let $V \nsubseteq X \backslash F$. Then there exists $y \in V \cap F$. For each $z \in W$, we have $y * z \in V * W \subseteq F$. Since $y \in F$ and $F$ is a filter, $z \in F$. Hence $W \subseteq F$ and so $x \in F$ which is a contradiction.

In Theorem 4.9 we will prove that the converse of Theorem 4.5 is also true.

Theorem 4.6. Let $(X, *, \tau)$ be a topological BE-algebra. If $1 \in \bigcap_{U \in \tau} U$, then $B \subseteq X$ is open if and only if 1 is an interior point of $B$. 
Proof. If $B$ is open, clearly, 1 is an interior point of $B$. Let 1 be an interior point of $B$ and $x * x=1$, there is an open neighborhood $V$ of 1 such that $x * x=1 \in V \subseteq B$. Since $*$ is continuous, there exists an open set $W$ containing $x$ such that $W * W \subseteq V$. By hypothesis, $1 \in W$, and hence $x \in W \subseteq W * W \subseteq V \subseteq B$. This proves that $x$ is an interior point of $B$.

Theorem 4.7. Let $(X, *, \tau)$ be a topological BE-algebra and $F_{1}$ the least open set containing 1 . If $x \in F_{1}$, then $F_{1}$ is the least open set containing $x$.

Proof. Let $x \in F_{1}$ and $U$ be an open set such that $x \in U$. Since $1 * x=x \in U$, there exist open neighborhoods $V$ of 1 and $W$ of $x$ such that $V * W \subseteq U$. We have $1=x * x \in F_{1} * W \subseteq V * W \subseteq U$. Therefore $1 \in U$. Since $F_{1}$ is the least open set such that $1 \in F_{1}, F_{1} \subseteq U$.

Theorem 4.8. Let $(X, *, \tau)$ be a topological BE-algebra and $F_{1}$ the least open set containing 1 . Then $F_{1}$ is a filter of $X$.

Proof. Let $x, x * y \in F_{1}$. By Theorem 4.7, $F_{1}$ is the least open set containing $x$. Since $x * y \in F_{1}$, there exist open neighborhoods $U$ of $x$ and $V$ of $y$ such that $U * V \subseteq F_{1}$. Hence $y=1 * y \in F_{1} * V \subseteq U * V \subseteq F_{1}$ and therefore $y \in F_{1}$.

Theorem 4.9. Let $(X, *, \tau)$ be a topological BE-algebra and $F$ a filter in $X$. If $F$ is closed then $F$ is open.

Proof. Suppose that $F$ is closed filter but not open. By Theorem 4.4, 1 is not an interior point of $F$. Hence $F_{1} \nsubseteq \subseteq F$, where $F_{1}$ is the least open set such that $1 \in F_{1}$. If $(X \backslash F) \cap F_{1}=\emptyset$, then $F_{1} \subseteq F$. Hence $(X \backslash F) \cap F_{1} \neq \emptyset$. Since $(X \backslash F) \cap F_{1}$ is open, by Theorem 4.7, $(X \backslash F) \cap F_{1}=F_{1}$. Thus $F_{1} \subseteq X \backslash F$ and so $1 \in X \backslash F$ which is a contradiction.

\section{Comparsion $\tau$ AND $\tau_{\Lambda}$}

In this section we assume that $(X, *, \tau)$ is a topological BE-algebra and $1 \neq x \in X$. The least open set containing $x$ is denoted by $U_{x}$.

Lemma 5.1. If $x * y \notin F_{1}$, then $y \notin U_{x}$ and $x \notin U_{y}$.

Proof. Let $y \in U_{x}$. Then $\{x, y\} \subseteq U_{x}$. Since $x * y \in U_{x * y}$, there exist open neighborhoods $V_{1}$ of $x$ and $V_{2}$ of $y$ such that $V_{1} * V_{2} \subseteq U_{x * y}$. We have $y \in U_{x} \subseteq V_{1}, y \in U_{y} \subseteq V_{2}$ and then $1=y * y \in U_{x} * U_{y} \subseteq U_{x * y}$. Put $z=x * y$. Since $z * z=1 \in F_{1}$, there exist open neighborhoods $W_{1}, W_{2}$ of $z$ such $W_{1} * W_{2} \subseteq F_{1}$. Then $1 * z \in U_{z} * U_{z} \subseteq W_{1} * W_{2} \subseteq F_{1}$. Hence $x * y=z \in F_{1}$ which is a contradiction. Similarly, we can show that $x \notin U_{y}$.

Theorem 5.1. Let $(X, *, \tau)$ be a topological BE-algebra and $\tau_{F_{1}}$ be the uniform topology induced by filter $F_{1}$. Then $\tau$ is finer than $\tau_{F_{1}}$. 
Proof. We will show that $U_{F_{1}}[x]=\bigcup_{y \in F_{1}[x]} U_{y}$ for all $x \in X$. Let $y \in U_{F_{1}[x]}$ and $z \in U_{y}$. If $z * y \notin F_{1}$ or $y * z \notin F_{1}$, then by Lemma 5.1, $z \notin U_{y}$. Thus $z * y \in F_{1}$ and $y * z \in F_{1}$. By $(6),(x * y) *((y * z) *(x * z))=1 \in F_{1}$. Since $x * y \in F_{1}$, we have $(y * z) *(x * z) \in F_{1}$ and so $x * z \in F_{1}$ because $y * z \in F_{1}$. Similarly, we can show that $z * x \in F_{1}$. Hence $z \in U_{F_{1}}[x]$. Therefore $U_{y} \subseteq U_{F_{1}}[x]$ for all $y \in U_{F_{1}}[x]$ and so $\bigcup_{y \in F_{1}[x]} U_{y} \subseteq U_{F_{1}}[x]$. It is clear that $U_{F_{1}}[x] \subseteq \bigcup_{y \in F_{1}[x]} U_{y}$.

Theorem 5.2. Let $(X, *, \tau)$ be a topological BE-algebra and $\tau_{F_{1}}$ be a uniform topology induced by filter $F_{1}$. If there exists $U \in \tau$ such that $U \notin \tau_{F_{1}}$, then there exist $x \in U$ and $y \in U_{F_{1}}[x]$ such that $y \notin U$ and the following properties holds:

(i) $x, y \notin F_{1}$.

(ii) $a * y \notin U_{F_{1}}[x] \cap U$, for all $a \in F_{1}$.

(iii) If $d \in U_{F_{1}}[x] \cap U$, then $a * d \neq y$, for all $a \in F_{1}$.

Proof. ( $i$ ) If $x \in F_{1}$, then by Theorem 4.7, $F_{1} \subseteq U$. Since $x \in F_{1}, y \in U_{F_{1}}[x]$ and $F_{1}$ is a filter, we have $y \in F_{1} \subseteq U$ which is a contradiction. Let $y \in F_{1}$. Since $y \in U_{F_{1}}[x]$ and $F_{1}$ is a filter, then $x \in F_{1}$ which is a contradiction.

(ii) Suppose that there exists some $a \in F_{1}$ such that $a * y \in U_{F_{1}}[x] \cap U$. There exist open neighborhoods $V_{1}$ of $a$ and $V_{2}$ of $y$ such that $V_{1} * V_{2} \subseteq U_{F_{1}}[x] \cap U$. By Theorem 4.7, $F_{1} \subseteq V_{1}$. Then $y=1 * y \in F_{1} * V_{2} \subseteq U_{F_{1}}[x] \cap U$. Hence $y \in U_{F_{1}}[x] \cap U$ which is a contradiction.

(iii) Suppose that there exists $a \in F_{1}$ such that $a * d=y$ for some $d \in$ $U_{F_{1}}[x] \cap U$. Since $1 * d=d \in U_{F_{1}}[x] \cap U$. there exist open neighborhoods $V_{1}$ of 1 and $V_{2}$ of $d$ such that $V_{1} * V_{2} \subseteq U_{F_{1}}[x] \cap U$. Then $y=a * d \in F_{1} * V 2 \subseteq$ $V_{1} * V_{2} \subseteq U_{F_{1}}[x] \cap U$. Hence $y \in U_{F_{1}}[x] \cap U$ which is a contradiction.

Lemma 5.2. Let $(X, *, \tau)$ be a topological BE-algebra and $\tau_{F_{1}}$ be the uniform topology induced by filter $F_{1}$. If $\tau_{F_{1}} \varsubsetneqq \tau$, then there exists $\emptyset \neq U \in \tau$ such that $U \varsubsetneqq U_{F_{1}}[x]$ for some $x \in X \backslash F_{1}$.

Proof. If $\tau_{F_{1}} \varsubsetneqq \tau$, then there exists $V_{1} \in \tau$ such that $V_{1} \notin \tau_{F_{1}}$. By definition of uniform topology, there exists $x \in V_{1}$ such that $U_{F_{1}}[x] \nsubseteq V_{1}$. Hence $U_{F_{1}}[x] \cap V_{1} \varsubsetneqq U_{F_{1}}[x]$. Put $U=U_{F_{1}}[x] \cap V_{1}$. Then $U \in \tau$ and $U \varsubsetneqq U_{F_{1}}[x]$. Suppose that $x \in F_{1}$. Then $U_{F_{1}}[x]=F_{1}$. Hence $x \in U$. By Theorem 4.7, $U_{F_{1}}[x]=F_{1} \subseteq U$ which is a contradiction.

Theorem 5.3. Let $\{0, a, b, 1\}$ be a bounded BE-algebra and let $(X, *, \tau)$ be a topological bounded BE-algebra and $\tau_{F_{1}}$ be the uniform topology induced by filter $F_{1}$. Then $\tau=\tau_{F_{1}}$.

Proof. Case 1. If $F_{1}=\{1\}$ or $F_{1}=X$, then it is clear that $\tau=\tau_{F_{1}}$.

Case 2. Suppose that $F_{1}=\{x, 1\}$ where $x \in\{a, b\}$ and $\tau_{F_{1}} \varsubsetneqq \tau$. Without less of generality, we assume that $F_{1}=\{a, 1\}$. By Lemma 5.3, there exists $U \in \tau$ such that $U \varsubsetneqq U_{F_{1}}[y]$ for some $y \in X \backslash F_{1}=\{0, b\}$. If $U_{F_{1}}[y]=\{y\}$, 
then $U=\emptyset$ which is a contradiction. So $U_{F_{1}}[y]=U_{F_{1}}[0]=U_{F_{1}}[b]=\{0, b\}$. Hence $b * 0 \in F_{1}$ and $0 * b \in F_{1}$. Then $b * 0=a$. If $a * 0 \in F_{1}$, then $0 \in F_{1}$ and $F_{1}=X$ which is a contradiction. Hence $a * 0=b$. Therefore $U=\{0\}$ or $U=\{b\}$. Consider the following cases:

(1) Suppose that $U=\{0\}$. Since $1 * 0=0 \in U$, there exist $V, W \in \tau$ such that $1 \in V, 0 \in W$ and $V * W \subseteq U$. So

$$
\{0, b\}=\{1 * 0, a * 0\} \subseteq F_{1} * U \subseteq V * W \subseteq U,
$$

which is a contradiction.

(2) Suppose that $U=\{b\}$. Since $a * 0=b \in U$, there exist $V, W \in \tau$ such that $a \in V, 0 \in W$ and $V * W \subseteq U$. By Theorem 4.7, $F_{1} \subseteq V$ and hence

$$
\{0, b\}=\{1 * 0, a * 0\} \subseteq F_{1} * W \subseteq V * W \subseteq U,
$$

which is a contradiction.

Case 3. Suppose that $F_{1}=\{a, b, 1\}$ and $\tau_{F_{1}} \subseteq \tau$. By Lemma 5.2, there exists $U \in \tau$ such that $\emptyset \neq U \varsubsetneqq U_{F_{1}}[y]$ for some $y \in X \backslash F_{1}$. Therefore $U \varsubsetneqq U_{F_{1}}[0]=\{0\}$ which is a contradiction.

Hence $\tau_{F_{1}}=\tau$ for all cases.

Lemma 5.3. Let $X=\{0, x, y, z, 1\}$ be a bounded BE-algebra and $F=\{x, 1\}$ be a filter of $X$. Then $y * z \neq x$ or $z * y \neq x$.

Proof. Let $y * z=z * y=x$. Then $x *(y * z)=x *(z * y)=x * x=1$. Consider following cases:

(1) Suppose that $x * y=0$. Since $1=x *(z * y)=z *(x * y)$, we get $z * 0=1$ and so $z \leq 0$. Since $0 \leq z$, we have $z=0$ which is a contradiction.

(2) Suppose that $x * y=y$. Since $1=x *(z * y)=z *(x * y)$, we have $z * y=1$ which is a contradiction.

(3) Suppose that $x * y=z$. Since $y *(x * y)=1$, we have $y * z=1$ which is contradiction.

(4) If $x * y=x$ or $x * y=1$, then $y \in F$ which is a contradiction.

Lemma 5.4. Let $X=\{0, x, y, z, 1\}$ be a bounded BE-algebra and $F=\{x, 1\}$ be a filter of $X$. Then

(i) if $U_{F}[y]=\{0, y\}$, then $x * 0=y$,

(ii) if $U_{F}[y]=\{y, z\}$, then $x * y=z$ or $x * z=y$,

(iii) if $U_{F}[y]=\{0, y, z\}$, then $(x * y=z$ and $x * 0=z)$ or $(x * z=y$ and $x * 0=y)$.

Proof. (i) Let $U_{F}[y]=\{0, y\}$, where $y \neq 0$. Then $y * 0 \in F$ and $0 * y \in F$. Therefore $y * x=x$. If $x * 0 \in F$, then $0 \in F$ which is a contradiction. Hence $x * 0=z$ or $x * 0=y$. Now, let $x * 0=z$. Then $z *(x * 0)=z * z=1$ and 
hence $x *(z * 0)=1$. Therefore $x \leq z * 0$. Since $F$ is a filter, $z * 0 \in F$. Also $0 * z=1 \in F$. Hence

$$
\begin{aligned}
& (z * 0) *((0 * y) *(z * y))=1 \\
& (y * 0) *((0 * z) *(y * z))=1 .
\end{aligned}
$$

Therefore $z * y \in F$ and $y * z \in F$ and so $z \in U_{F}[y]$ which is a contradiction. Hence $x * 0=y$.

(ii) By Lemma 5.3, $y * z=1, z * y=x$ or $y * z=x, z * y=1$. Let $y * z=1, z * y=x$. Hence $y \leq z$ and $z \leq x * y$ because $x *(z * y)=x * x=1$ hence $z *(x * y)=1$ and so $z \leq x * y$. If $x * y \in F$, then $y \in F$ which is a contradiction. Since $0 \leq y \leq z \leq x * y$ and $x * y \notin F$, then $x * y=z$. Similarly, if $y * z=x, z * y=1$, then $x * z=y$.

(iii) Let $U_{F}[y]=\{0, y, z\}$. Then $y * 0=x, z * 0=x, y * z \in F$ and $z * y \in F$. By Lemma 5.3, $y * z=1, z * y=x$ or $y * z=x, z * y=1$. If $y * z=1$ and $z * y=x$, then $x * y=z$ similar to part (2). Since $y \leq z \leq x * 0$ and $x * 0 \notin F$, then $x * 0=z$. If $y * z=x$ and $z * y=1$, then $x * z=y$ similar to part (2). Since $z \leq y \leq x * 0$ and $x * 0 \notin F$, then $x * 0=y$.

Theorem 5.4. Let $X$ be a bounded BE-algebra where $|X|=5$. If $(X, *, \tau)$ is a topological BE-algebra and $F=\{x, 1\}$ is a filter in $X$, then $\tau=\tau_{F}$.

Proof. Suppose that $\tau \neq \tau_{F}$. By Lemma 5.2, there exists $U \in \tau$ such that $U \varsubsetneqq U_{F}[y]$ for some $y \in X \backslash F$. Consider the following cases:

Case (1). $U_{F}[y]=\{y\}$. Then $U=\emptyset$.

Case (2). $U_{F}[y]=\{0, y\}$, where $y \neq 0$. By Lemma 5.4 part (1), $x * 0=y$. Since $U \varsubsetneqq U_{F}[y]$, then $U=\{0\}$ or $U=\{y\}$.

(1) Suppose that $U=\{0\}$. Since $1 * 0=0 \in U$, there exist $V, W \in \tau$ such that $1 \in V, 0 \in W$ and $V * W \subseteq U$. Then we have

$$
\{0, y\}=\{1 * 0, x * 0\} \subseteq F * U \subseteq V * W \subseteq U .
$$

Hence $y \in U$, which is a contradiction.

(2) Suppose that $U=\{y\}$. Since $x * 0=y$, then there exist $V, W \in \tau$ such that $x \in V, 0 \in W$ and $V * W \subseteq U$. Then we have

$$
\{0, y\}=\{1 * 0, x * 0\} \subseteq F * W \subseteq V * W \subseteq U .
$$

Hence $0 \in U$, which is a contradiction.

Case (3). Suppose that $U_{F}[y]=\{y, z\}$, where $y, z \neq 0$. By Lemma 5.3, $y * z=1, z * y=x$ or $y * z=x, z * y=1$. Let $y * z=1, z * y=x$. Then by Lemma 5.4 part $(2), x * y=z$. Since $U \varsubsetneqq U_{F}[y]$, we have $U=\{y\}$ or $U=\{z\}$.

(1) Suppose that $U=\{y\}$. Since $1 * y=y \in U$, there exist $V, W \in \tau$ such that $1 \in V, y \in W$ and $V * W \subseteq U$.

$$
\{y, z\}=\{1 * y, x * y\} \subseteq F * U \subseteq V * W \subseteq U,
$$

which is a contradiction. 
(2) Suppose that $U=\{z\}$. Since $x * y=z \in U$, there exist $V, W \in \tau$ such that $x \in V, y \in W$ and $V * W \subseteq U$.

$$
\{y, z\}=\{1 * y, x * y\} \subseteq F * W \subseteq V * W \subseteq U,
$$

which is a contradiction.

Case (4). Suppose that $U_{F}[y]=\{0, y, z\}$. By Lemma 5.3, $y * z=1, z * y=x$ or $y * z=x, z * y=1$. Let $y * z=1$ and $z * y=x$. By Lemma 5.4 part (3), $x * y=z$ and $x * 0=z$. Then

(1) Suppose that $U=\{0\}$. Since $1 * 0=0 \in U$, there exist $V, W \in \tau$ such that $1 \in V, 0 \in W$ and $V * W \subseteq U$.

$$
\{0, z\}=\{1 * 0, x * 0\} \subseteq F * U \subseteq V * W \subseteq U=\{0\},
$$

which is a contradiction.

(2) Suppose that $U=\{y\}$. Since $1 * y=y \in U$, there exist $V, W \in \tau$ such that $1 \in V, y \in W$ and $V * W \subseteq U$.

$$
\{y, z\}=\{1 * y, x * y\} \subseteq F * U \subseteq V * W \subseteq U,
$$

which is a contradiction.

(3) Suppose that $U=\{z\}$. Since $x * y=z \in U$, there exist $V, W \in \tau$ such that $x \in V, y \in W$ and $V * W \subseteq U$.

$$
\{y, z\}=\{1 * y, x * y\} \subseteq F * W \subseteq V * W \subseteq U,
$$

which is a contradiction.

(4) Suppose that $U=\{0, z\}$. Since $x * y=z \in U$, there exist $V, W \in \tau$ such that $x \in V, z \in W$ and $V * W \subseteq U$.

$$
\{y, z\}=\{1 * y, x * y\} \subseteq F * W \subseteq V * W \subseteq U,
$$

which is a contradiction.

(5) Suppose that $U=\{0, y\}$. Since $1 * y=y \in U$, there exist $V, W \in \tau$ such that $1 \in V, y \in W$ and $V * W \subseteq U$.

$$
\{y, z\}=\{1 * y, x * y\} \subseteq F * W \subseteq V * W \subseteq U,
$$

which is a contradiction.

(6) Suppose that $U=\{y, z\}$. Since $x * 0=z \in U$, then there exist $V, W \in \tau$ such that $x \in V, 0 \in W$ and $V * W \subseteq U$.

$$
\{0, z\}=\{1 * 0, x * 0\} \subseteq F * W \subseteq V * W \subseteq U,
$$

which is a contradiction.

Hence $\tau=\tau_{F}$.

Theorem 5.5. Let $X$ be a bounded BE-algebra where $|X|=5$. If $(X, *, \tau)$ is a topological BE-algebra, then $\tau=\tau_{F_{1}}$. 
Proof. Case (1). If $F_{1}=\{1\}$ or $F_{1}=X$, then it is clear $\tau=\tau_{F_{1}}$.

Case (2). If $F_{1}=\{x, 1\}$, then $\tau=\tau_{F_{1}}$ by Theorem 5.4.

Case (3). Suppose that $F_{1}=\{z, x, 1\}$ but $\tau \neq \tau_{F_{1}}$. By Lemma 5.2, there exists $U \in \tau$ such that $U \varsubsetneqq U_{F_{1}}[a]$ for some $a \in X \backslash F_{1}=\{0, y\}$. Then

(i) If $U_{F_{1}}[a]=\{a\}$, then $U=\emptyset$.

(ii) If $U_{F_{1}}[a]=U_{F_{1}}[y]=\{0, y\}$, then $y \leq x * 0$. Since $x * 0 \notin F_{1}$, thus $x * 0=y$. Since $U \varsubsetneqq U_{F_{1}}$, we have $U=\{y\}$ or $U=\{0\}$.

(1) Suppose that $U=\{y\}$. Since $x * 0=y \in U$, there exist $V, W \in \tau$ such that $x \in V, 0 \in W$ and $V * W \subseteq U$.

$$
\{0, y\} \subseteq\{1 * 0, x * 0\} \subseteq F * W \subseteq V * W \subseteq U,
$$

which is a contradiction.

(2) Suppose that $U=\{0\}$. Since $1 * 0=0 \in U$, there exist $V, W \in \tau$ such that $1 \in V, 0 \in W$ and $V * W \subseteq U$.

$$
\{0, y\}=\{1 * 0, x * 0\} \subseteq F_{1} * U \subseteq V * W \subseteq U,
$$

which is a contradiction.

Case (4). If $F_{1}=\{z, y, x, 1\}$, then $\tau_{F_{1}}=\tau$ by Lemma 5.2 .

\section{REFERENCES}

[1] S. S. Ahn, Y. H. Kim and J. M. Ko, Filters in commutative BE-algebras, Commun Korean Math. Soc. 27 (2012), No. 2.

[2] Z. Ciloglu and Y. Ceven, Commutative and bounded BE-algebras, Hindawi Publishing Corporation Algebra Volume 2013, Article ID 473714, 5 pages.

[3] K. Iséki, On BCI-algebras, Math. Seminar Notes, 8(1980), 125-130.

[4] K. Iséki and Tanaka, An introduction to theory of BCK-algebras, Math. Japonica, 23(1978), 1-26.

[5] K.D. Joshi, Introduction to general topology, New Age International Publisher, India, 1997.

[6] Y. B. Jun, E. H. Roh and H. S. Kim, On BH-algebras, Sci. Math. Japonica Online, 1(1998), 347-354.

[7] H. S. Kim and Y. H. Kim, On BE-algebras, Scientiae Mathematicae Japonica Online, e-2006, 1299-1302.

[8] M. Mohamadhasani and M. Haveshki, Some clopen setes in uniform topology on BE-algebra, Scientia Magna Vol. 6 (2010), No. 4, 85-91.

[9] J. Neggers and H. S. Kim, On d-algebra, Math. Slovaca, 49 (1999), 19-26.

[10] A. Walendziak, On commutative BE-algebras, Scientiae Mathematicae Japonica Online, (e-2008), 585-588. 
[11] A. Walendziak, On normal filters and congruence relations in BE-algebras, Commentationes Mathematicae Vol. 52, (2012), 199-205.

[12] Y. H. Yon, S. M. Lee and K. H. Kim, On congruences and BE-relations in BEalgebras, International Mathematical Forum, 5, (2010), 2263-2270.

\section{S. Mehrshad}

FaCUlty of SCIENCES

UNIVERSITY OF ZABOL

IRAN

E-mail address: smehrshad@uoz.ac.ir

J. Golzarpoor

FaCULTY of SCIENCES

UNIVERSITY OF ZABOL

IRAN

E-mail address: javad-golzarpoor@uoz.ac.ir 